\title{
BMJ
}

\section{Migraines during pregnancy linked to stroke and vascular diseases: US population based case-control study}

\author{
Cheryl D Bushnell, associate professor, ${ }^{1}$ Margaret Jamison, statistician and head of program evaluations, ${ }^{2}$ \\ Andra $\mathrm{H}$ James, assistant professor ${ }^{3}$
}

${ }^{1}$ Department of Neurology, Wake Forest University Health Sciences, Medical Center Boulevard, Winston-Salem, NC 27157, USA

${ }^{2}$ University of North Carolina School of Medicine, Office of Educational Development, Chapel Hill, NC 27599-7530 jamisonm@earthlink.net

${ }^{3}$ Division of Maternal FetalMedicine, Department of

Obstetrics and Gynecology, Duke University Medical Center, Durham, NC 27710 Andra. james@duke.edu

Correspondence to: C D Bushnell cbushnel@wfubmc.edu

Cite this as: BMJ 2009:338:b664 doi:10.1136/bmj.b664

\section{ABSTRACT}

Objective To examine the association between migraine and cardiovascular diseases during pregnancy.

Design US population based case-control study.

Setting Nationwide inpatient sample, from the Healthcare Cost and Utilization Project of the Agency for Healthcare Research and Quality.

Population 18345538 pregnancy related discharges from 2000 to 2003.

Main outcome measures Diagnosis of migraine, as identified by ICD-9 codes 346.0 and 346.1. Stroke and other vascular diseases were identified by using standard ICD-9 codes.

Results From the hospital discharges with a pregnancy discharge code, 33956 migraine codes were identified: 185 per 100000 deliveries. Diagnoses that were jointly associated with migraine codes during pregnancy (excluding pre-eclampsia) were stroke (odds ratio 15.05, $95 \%$ confidence interval 8.26 to 27.4 ), myocardial infarction/heart disease $(2.11,1.76$ to 2.54$)$, pulmonary embolus/venous thromboembolism (3.23, 2.06 to 7.07 ), and hypertension $(8.61,6.43$ to 11.54$)$, as well as preeclampsia/gestational hypertension (2.29, 2.13 to 2.46), smoking $(2.85,2.53$ to 3.21$)$, and diabetes $(1.96,1.64$ to 2.35). However, migraine was not associated with several non-vascular diagnoses (pneumonia, transfusions, postpartum infection or haemorrhage).

Conclusions In this large, population based sample of pregnant women admitted to hospital, a strong relation existed between active peripartum migraine and vascular diagnoses during pregnancy. Because these data do not allow determination of which came first, migraine or the vascular condition, prospective studies of pregnant women are needed to explore this association further.

\section{INTRODUCTION}

Migraine headache is a neurovascular headache disorder that occurs in $11 \%$ to $26 \%$ of women of childbearing age. ${ }^{12}$ The one year prevalence of migraine peaks at 33\% in women aged between 35 and 39. Although migraine is very common in this age group, only a few studies have focused on the prevalence of migraine during pregnancy. ${ }^{45}$ The results of natural history studies have suggested that migraines generally improve during pregnancy, although these studies vary widely. ${ }^{6}$

The conditions associated with active or worsening peripartum migraines are poorly understood. That migraine may be associated with pre-eclampsia, one of the hypertensive disorders of pregnancy, has recently been recognised. ${ }^{7-10}$ Using a population based analysis of pregnancy related discharges from 2000-1, we found that migraine was associated with a 17 -fold increased risk of pregnancy related stroke and a fourfold increased risk of acute myocardial infarction. ${ }^{112}$ In addition, migraine has been increasingly associated with stroke and cardiovascular disease in older patients outside of pregnancy, although this is most noticeable in migraine with aura. ${ }^{13-15}$ The objective of the analysis reported here was to determine the prevalence of peripartum migraine headache and its associated medical conditions and complications during pregnancy by using a population based analysis of pregnancy related discharges from hospital in the United States in 2000-3.

\section{METHODS}

The nationwide inpatient sample, from the Healthcare Cost and Utilization Project of the Agency for Healthcare Research and Quality, contains data from five to eight million hospital stays from approximately 1000 hospitals and is the largest all-payer inpatient care database in the United States. ${ }^{1617}$ The nationwide inpatient sample is a $20 \%$ stratified sample of all discharges and allows for national estimates. It includes information derived from typical discharge abstracts. This information contains primary and secondary discharge diagnoses, with the corresponding codes, determined from the description in the discharge summary. In addition, these records have safeguards to protect the privacy of individual patients, physicians, and hospitals. Data are available for the purposes of aggregate statistical reporting, analysis, and research. ${ }^{1617}$ Although the data are limited, the nationwide inpatient sample is the most reliable source of data on hospital admissions and discharges. Reliability is supported by agreement between the sample, a telephone survey, and the national health interview survey (a national door to door survey). Invalid or 
Table 1 Timing of pregnancy related admission to hospital with diagnosis of migraine

\begin{tabular}{lccc}
$\begin{array}{l}\text { Timing of admission during } \\
\text { pregnancy }\end{array}$ & $\begin{array}{c}\text { Migraine rate per } 100000 \\
\text { discharges }\end{array}$ & Odds ratio $(95 \% \mathrm{Cl})$ & P value \\
Vaginal delivery & 117 & 1.0 & Referent \\
\hline Caesarean delivery & 150 & $1.3(1.2$ to 1.4$)$ & $<0.001$ \\
\hline Antepartum (abortion) & 231 & $2.0(1.6$ to 2.4$)$ & $<0.001$ \\
\hline Antepartum (no abortion) & 1049 & $9.0(8.2$ to 9.9$)$ & $<0.001$ \\
\hline Postpartum & 665 & $5.7(4.8$ to 6.7$)$ & $<0.001$ \\
\hline
\end{tabular}

inconsistent diagnostic codes are flagged. ${ }^{1617}$ All data are anonymised, so informed consent was waived.

We used the ICD-9 (international classification of diseases, 9th revision) to identify records in the nationwide inpatient sample for all pregnancy related discharge codes for the years 2000-3 (the latest data available at the start of the study). We classified the pregnancy related discharge records included in the sample from that time period as to whether they were an antepartum admission, an admission at the time of delivery, or a postpartum admission. We defined an antepartum admission as any discharge record with a pregnancy related code (ICD-9 codes 630-648) that did not also include a delivery code. We defined an admission for delivery as any discharge record that included a delivery code (ICD-9 codes 74 for caesarean delivery and 72, 73, 75, v27, or 650-659 for vaginal delivery). We defined a postpartum admission as any discharge record that included a postpartum diagnosis (ICD-9 codes 660-677) and did not also include a delivery code.

We used standard ICD-9 codes 346.10 (common migraine without aura, not intractable), 346.11 (common migraine, intractable), 346.00 (migraine with aura, not intractable), 346.01 (migraine with aura, intractable), and 346.9 (migraine unspecified) to identify diagnoses of migraine. ${ }^{18}$ We excluded tension headache (ICD-9 code 784) from this analysis. We classified strokes as subarachnoid haemorrhage (ICD-9 430), intracerebral haemorrhage (ICD-9 code 431), ischaemic event (ICD-9 codes 434 and 436), cerebral venous thrombosis (ICD-9 code 325), or

Table 2|Peripartum diagnosis of migraine by age at discharge and race/ethnicity

\begin{tabular}{lccc} 
& $\begin{array}{c}\text { Migraine rate per } \mathbf{1 0 0} 000 \\
\text { discharges }\end{array}$ & Odds ratio $(95 \% \mathrm{Cl})$ & P value \\
\hline $\begin{array}{l}\text { Age group (years) } \\
\langle 20\end{array}$ & 96 & 1.0 & Referent \\
\hline $20-24$ & 168 & $1.8(1.6$ to 2.0$)$ & $<0.001$ \\
\hline $25-29$ & 231 & $2.1(1.9$ to 2.4$)$ & $<0.001$ \\
\hline $30-34$ & 205 & $2.1(1.9$ to 2.4$)$ & $<0.001$ \\
\hline $35-39$ & 218 & $2.3(2.0$ to 2.6$)$ & $<0.001$ \\
\hline$\geq 40$ & 228 & $2.4(2.0$ to 2.9$)$ & $<0.001$ \\
\hline Race/ethnicity & & 1.0 & $<$ Referent \\
\hline White & 245 & $0.7(0.6$ to 0.8$)$ & $<0.001$ \\
\hline African-American & 175 & $0.4(0.3$ to 0.4$)$ & $<0.001$ \\
\hline Hispanic & 85 & $0.8(0.7$ to 0.9$)$ & - \\
\hline Other & 86 & - & \\
\hline Missing & 191 & & \\
\hline
\end{tabular}

pregnancy related cerebrovascular event (ICD-9 code 674.0). ${ }^{18}$ Although several ICD-9 codes encompass ischaemic stroke, we used 434 and 436 because review of medical records showed them to have the highest sensitivity and specificity. ${ }^{19}$

For other vascular diagnoses, we used the ICD-9 codes for myocardial infarction (410), heart disease (648.5, 648.6), pulmonary embolus (673.2, 415.1), deep venous thrombosis $(671.3,451.1,451.2)$, and thrombophilia $(282.62,282.42,282.64) .{ }^{18}$ Additional ICD-9 codes included hypertension (401-405), diabetes $(648.0,250)$, smoking (305.1, V15.82), gestational hypertension and pre-eclampsia $(642.0,642.2$ 642.7), gestational diabetes (648.8), cardiomyopathy (425), pulmonary hypertension (416.8), anaemia (648.2, 280, 281), pneumonia (480-483, 486-487), transfusion $(99.02,99.04,99.00,99.07)$, postpartum infection $(670,672)$, postpartum haemorrhage $(666$, 669.1), and intrauterine fetal death (656.4).

\section{Statistical analysis}

We weighted data by the strata, sampling units (hospitals), and sampling weights based on the nationwide inpatient sample sampling design. We used Stata 8.0 and the SVY (survey data) commands with these weights for both descriptive and inferential analyses.

We used methods accounting for the survey design to approximate counts and standard errors. We calculated frequencies of migraine discharges for each age group, race/ethnicity, timing of pregnancy related discharge, comorbidity, and complication of pregnancy by using two way weighted $\chi^{2}$ analyses. To determine which of these factors were associated with discharge codes for peripartum migraine, we estimated prevalence odds ratios, $95 \%$ confidence intervals, and $\mathrm{P}$ values from logistic regression analyses in accordance with the sampling design. We identified jointly associated factors with multivariable logistic regression modelling developed from statistically $(\mathrm{P}<0.05)$ and clinically significant common vascular comorbidities. As pre-eclampsia diagnoses were significantly correlated with other vascular event diagnoses, this may have confounded the associations with migraine and other vascular diagnoses. Therefore, for records with a diagnosis of pre-eclampsia in addition to other vascular diagnoses, we excluded the pre-eclampsia codes and created new variables for stroke, venous thromboembolism/pulmonary embolus, and heart disease/ acute myocardial infarction. We then entered these new variables into a logistic regression model that was adjusted for age but also with the influence of preeclampsia removed in order to determine the independent associations between migraine and vascular diagnoses.

\section{RESULTS}

During the period from 2000 to 2003, 18345538 discharges with pregnancy codes occurred. Among the pregnancy related discharges, 33956 discharges with a diagnosis of migraine occurred-185 per 100000 deliveries. As shown in table 1, most discharges with 
Table $3 \mid$ Association between peripartum migraine and vascular diagnoses or cardiovascular risk factors

\begin{tabular}{|c|c|c|c|c|}
\hline Vascular diagnosis or risk factor & ICD-9 codes & $\begin{array}{l}\text { Migraine rate } \\
\text { per } 100000^{*}\end{array}$ & $\begin{array}{l}\text { Odds ratio } \\
(95 \% \mathrm{Cl})\end{array}$ & $P$ value \\
\hline All strokes & $\begin{array}{c}430,431,434 \\
436,325,674.0\end{array}$ & 2839 & $\begin{array}{l}15.8(11.1 \text { to } \\
22.5)\end{array}$ & $<0.001$ \\
\hline Ischaemic stroke & 434,436 & 5364 & $\begin{array}{l}30.7(17.4 \text { to } \\
34.1)\end{array}$ & $<0.001$ \\
\hline Intracerebral haemorrhage & 431 & 1653 & 9.1 (3.0 to 27.8$)$ & $<0.001$ \\
\hline Cerebral venous thrombosis & 325 & 0 & - & - \\
\hline Subarachnoid haemorrhage & 430 & 615 & $3.4(0.5$ to 24.1$)$ & 0.23 \\
\hline $\begin{array}{l}\text { Pregnancy related cerebrovascular } \\
\text { event }\end{array}$ & 674 & 2546 & $\begin{array}{c}14.1(8.7 \text { to } \\
23.1)\end{array}$ & $<0.001$ \\
\hline Myocardial infarction & 410 & 1771 & $4.9(1.7$ to 14.2$)$ & $<0.001$ \\
\hline Heart disease & $648.5,648.6$ & 410 & 2.1 (1.8 to 2.6$)$ & $<0.001$ \\
\hline Pulmonary embolus & $673.2,415.1$ & 760 & $3.1(1.7$ to 5.6$)$ & $<0.001$ \\
\hline Deep venous thrombosis & $\begin{array}{c}671.3,451.1 \\
451.2\end{array}$ & 515 & $2.4(1.3$ to 4.2$)$ & $<0.001$ \\
\hline Thrombophilia & $\begin{array}{c}282.62,282.42 \\
282.64\end{array}$ & 781 & $3.6(2.1$ to 6.1$)$ & $<0.001$ \\
\hline Hypertension & $401-405$ & 740 & $3.6(3.1$ to 4.2$)$ & $<0.001$ \\
\hline Cardiomyopathy & 425 & 636 & $2.2(1.3$ to 3.7$)$ & $<0.001$ \\
\hline Diabetes & $648.0,250$ & 482 & 2.3 (1.9 to 2.7 ) & $<0.001$ \\
\hline Smoking & $305.1, \mathrm{~V} 15.82$ & 489 & 2.7 (2.4 to 3.1$)$ & $<0.001$ \\
\hline
\end{tabular}

ICD-9= international classification of diseases, 9th revision.

*Migraine rate per 100000 discharges for each vascular diagnosis or risk factor.

a diagnosis of migraine were coded at the time of delivery (antepartum without abortion, 1049 per 100000 discharges). The proportion of diagnoses of migraine increased with increasing maternal age (table 2). Women 40 years of age or older were 2.4fold (95\% confidence interval 2.0 to 2.9$)$ more likely to have migraine discharge codes than were women under 20 years of age, and white women were more likely to have migraine discharge codes than any other race/ethnicity (table 2).

We also found a strong association between migraine discharge codes and stroke codes of all types (odds ratio $15.8,95 \%$ confidence interval 11.1 to 22.5$)$. The strongest association was with ischaemic stroke (odds ratio $30.7,17.4$ to 34.1 ), whereas cerebral venous thrombosis and subarachnoid haemorrhage were not associated with migraine discharge codes (table 3). In addition, codes for myocardial infarction (odds ratio $4.9,1.7$ to 14.2$)$, heart disease $(2.1,1.8$ to 2.6 ), pulmonary embolus (3.1, 1.7 to 5.6$)$, deep venous thrombosis (2.4, 1.3 to 4.2$)$, and thrombophilia (3.6, 2.1 to 6.1 ) were all significantly associated with migraine discharge codes. Vascular risk factors were also associated with migraine codes; these included diabetes (odds ratio 2.3, 1.9 to 2.7), hypertension (3.6, 3.1 to 4.2 ), and cigarette smoking $(2.7,2.4$ to 3.1 ) (table 3 ).

Some, but not all pregnancy related complications were associated with migraine codes (table 4). For example, pre-eclampsia/gestational hypertension was associated (odds ratio 2.3, 2.1 to 2.5 ), but gestational diabetes was not (1.0, 0.9 to 1.1$)$. In addition, migraine was not associated with several non-vascular diagnoses, such as pneumonia, transfusions, postpartum infection or haemorrhage, or intrauterine fetal death (table 4). Hypertension codes exclusive of gestational hypertension/pre-eclampsia (odds ratio 11.9, 9.2 to 15.4) were more strongly associated with migraine codes than were the gestational hypertension/preeclampsia codes alone (2.2, 2.0 to 2.4) (table 5).

A multivariable logistic regression included age and separated the pre-eclampsia diagnoses from stroke, vascular diagnoses, hypertension, smoking, and diabetes. In addition, related diagnoses, such as heart disease and acute myocardial infarction or venous thromboembolism and pulmonary embolus were combined into one variable. As shown in table 6, increasing age (odds ratio $1.03,1.02$ to 1.03 ), preeclampsia $(2.29,2.13$ to 2.46$)$, stroke $(15.05,8.26$ to $27.4)$, venous thromboembolism/pulmonary embolus (3.23, 2.06 to 7.07$)$, heart disease/myocardial infarction $(2.11,1.76$ to 2.54$)$, smoking $(2.85,2.53$ to 3.21$)$, and diabetes $(1.96,1.64$ to 2.35$)$ were all independently associated with migraine discharge codes.

\section{DISCUSSION}

In this population based case-control study of pregnant women admitted to hospital, we found an association between codes for a diagnosis of migraine and codes for vascular events - especially ischaemic stroke - as well as vascular risk factors and pre-eclampsia. Pre-eclampsia, the most influential variable in relation to migraine, was removed in order to reduce confounding in the multivariable logistic regression model, which then showed the independent association between codes for migraine and those for stroke, heart disease, venous thromboembolism, and hypertension.

ICD-9 diagnoses of migraine $(0.185 \%)$ were uncommon during pregnancy related discharges in this study, but this may reflect only severe migraine episodes or generally intractable headaches occurring during hospital admissions as a primary or secondary diagnosis resulting in migraine being listed as a discharge code. However, migraine with aura was coded infrequently, which perhaps under-represents this important subset at risk of vascular disease. The prevalence of migraine codes may also be an underestimate because we could not determine the number of women who may have had a history of migraines before pregnancy but were not coded because their migraines were quiescent during pregnancy. In addition, this database may not have captured women with migraines who were managed exclusively as outpatients. In this analysis, based on the standard practice of coders using only the diagnoses listed by the discharging physician, we assumed that migraines were active during the pregnancy related admission in order to be included in the ICD-9 code list. On the basis of the select group of pregnant women with migraines coded during the hospital admission, this may not represent the population of women with migraine as a whole.

Of the associations between peripartum migraine codes and vascular diagnosis codes, the relation between migraine and stroke was the strongest. Even with the overlapping pre-eclampsia diagnosis codes 


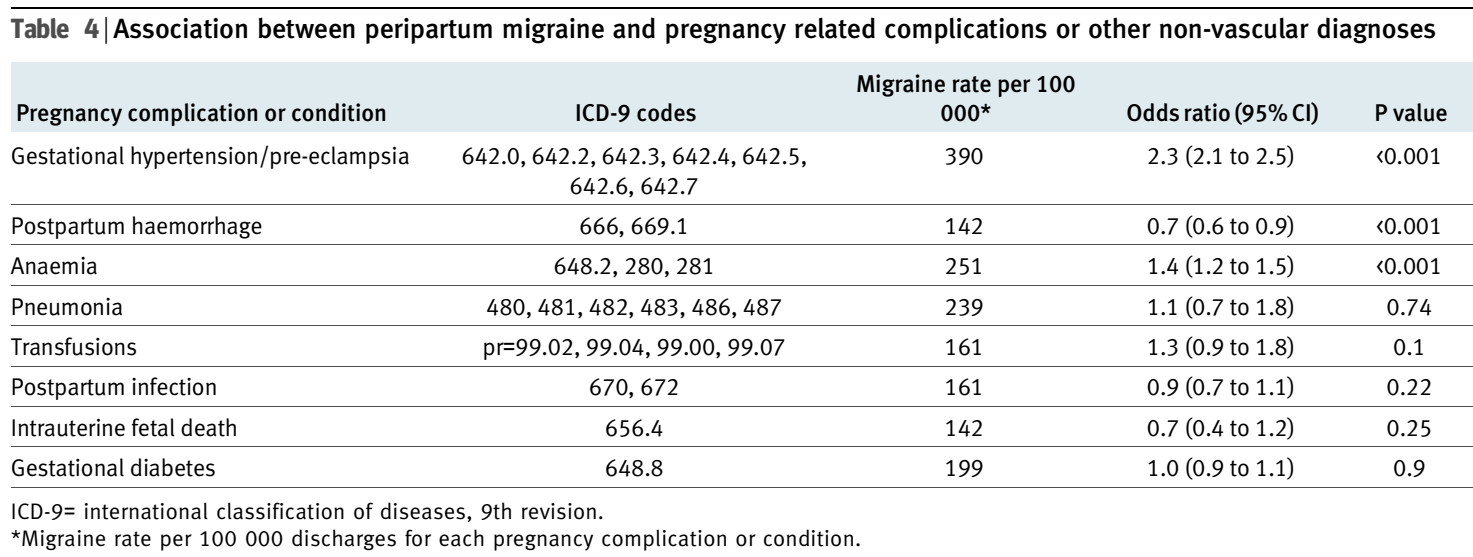

removed, we found a 15 -fold increased odds of stroke in women with peripartum migraine (odds ratio 15.1, $95 \%$ confidence interval 8.3 to 27.4 ). This is consistent with our previous analysis of the nationwide inpatient sample from the years 2000 and 2001, in which we found that the reverse was also true. ${ }^{11}$ In other words, when pregnancy related stroke was the primary outcome, we found a 17 -fold increased odds of having a diagnosis of migraine. These results in pregnant women are also consistent with studies of non-pregnant women in which the association seems to be most significant in younger women or those with migraine with aura. ${ }^{20-24}$ This association has also been shown in studies of older women. For example, in the Women's Health Study, healthy women with migraines with aura had a 70\% increased odds of ischaemic stroke, and the association was strongest in women aged 45 to 55 years. ${ }^{13}$ A recent meta-analysis also supports the association between migraine and stroke, again most significantly in people with migraine with aura. ${ }^{25}$

We also found important differences according to type of stroke. Ischaemic stroke was the type most strongly associated with migraine codes in our analysis, which is consistent with studies of non-pregnant women, such as the Women's Health Study. ${ }^{13}$ However, the positive relation between intracerebral haemorrhage and migraine codes is in contrast to the Women's Health Study, which reported no association with migraine. Only one other study found an association between migraine and intracerebral haemorrhage, but this was in non-pregnant women. ${ }^{21}$ Interestingly, subarachnoid haemorrhage was associated with migraine codes, but this did not reach significance (table 3). Clearly, larger numbers are needed to investigate this association further in pregnant women.

We also found that migraine was associated with acute myocardial infarction and heart disease during pregnancy. This is consistent with a previous analysis of pregnancy related acute myocardial infarction, which found a significant association with ICD-9 codes for migraine headache (odds ratio $4.2,1.0$ to 7.1 ). ${ }^{12}$ The only other published study that examined the relation between migraine and vascular diseases in pregnancy was a study that used data from the National
Collaborative Perinatal Project. This study, which focused on the possible confounding association between cigarette smoking and migraine during pregnancy, reported that pregnant migraineurs had a higher prevalence of heart disease than non-migraineurs, but that this was primarily in women who smoked cigarettes. ${ }^{26}$ One limitation of this study was that it included 512 pregnant migraineurs, whereas our cohort consisted of nearly 34000 .

In non-pregnant women, the association between migraine and coronary heart disease has been positive in some studies but not in others. ${ }^{1427-29}$ The Atherosclerosis Risk in Communities cohort study found no association between self reported migraine with or without aura and verified coronary heart disease events. ${ }^{28}$ However, a significant association was found with angina, as determined by the Rose angina questionnaire, suggesting that migraineurs might have a lower threshold for chest pain. ${ }^{28}$ The Kaiser Permanente Medical Care Program reported a similar association between migraine and chest pain but not myocardial infarction. ${ }^{29}$ However, the investigators for the Women's Health Study found that migraine with aura (but not migraine without aura) was associated with an increased risk of myocardial infarction (odds ratio $2.08,1.30$ to 3.31 ), major cardiovascular disease $(2.15,1.58$ to 2.92$)$, and ischaemic cardiovascular death $(2.33,1.21$ to 4.51$) .{ }^{14}$

Women with peripartum migraines were also more likely to have vascular risk factors, such as diabetes, hypertension, and tobacco smoking, which was consistent with the association with vascular events. Hypertension, in isolation from pregnancy related hypertension, was the vascular risk factor most strongly associated with migraine codes (table 5). Other analyses of non-pregnant migraineurs have shown a similar association between migraine and cardiovascular risk factors. In the Genetic Epidemiology of Migraine study, migraineurs had about a $40 \%$ increased odds of high blood pressure (odds ratio $1.38,1.0$ to 1.8$)$, were more likely to smoke cigarettes $(1.59,1.2$ to 2.2$)$, and were twice as likely to have a 10 year Framingham risk of coronary heart disease death or myocardial infarction of greater than $20 \%$ compared with non-migraineurs. ${ }^{7}$ The National 
Collaborative Perinatal Project also reported a correlation between migraine and smoking in pregnant women. ${ }^{26}$ In contrast, the most recent analysis of the Women's Health Study showed that women who had active migraine with aura and ischaemic stroke were younger (age 45 to 49) and had lower blood pressure, lower cholesterol, and a Framingham risk of less than $1 \%$, whereas those with active migraine with aura and myocardial infarction tended to have a Framingham risk of $10 \%$ or above. ${ }^{15}$ Overall, because of the trends for onset of obesity and cardiovascular risk factors at earlier ages, including the childbearing years, more research focused on migraine and cardiovascular risk during pregnancy is needed.

We found that women with pre-eclampsia were twice as likely to have peripartum migraine as those without pre-eclampsia. Of all of the diagnoses entered into the multivariable model, this relation was one of the strongest (as shown by a small standard error and a high t value in table 6). In fact, in order to examine the associations between migraine and other vascular diagnoses, the impact of pre-eclampsia had to be eliminated because of confounding. This finding is consistent with several other studies in the literature. In the Genetic Epidemiology of Migraine study, women with migraine of any type were $60 \%$ more likely to have a history of gestational hypertension than were those without migraine (odds ratio $1.63,1.2$ to 2.1 ). ${ }^{7} \mathrm{~A}$ history of pre-pregnancy migraines may also increase the likelihood of a woman developing severe preeclampsia. ${ }^{930}$ Because severe migraines during pregnancy have been linked to a higher risk of developing pre-eclampsia, ${ }^{10}$ differentiating peripartum migraines from the headaches related to severe pre-eclampsia needs to be pursued in future studies.

Diagnoses of migraine were also associated with venous thromboembolism and thrombophilia. Similarly, the National Collaborative Perinatal Project reported a significant association between migraines and thrombosis, but this was primarily in pregnant women who were smokers. ${ }^{26}$ Several studies have investigated the possibility of a heightened hypercoagulable state in migraineurs, such as high antiphospholipid antibodies, ${ }^{31}$ platelet hyperaggregability, or hereditary markers of hypercoagulability. ${ }^{32}$ However, these studies have too many methodological shortcomings (small sample sizes, a wide variety of non-standardised assays) to allow conclusions about a definitive hypercoagulable state related specifically to migraine to be made. ${ }^{32}$ Interestingly, a recent study also showed that migraineurs aged 55 to 94 were significantly more likely to have a lifetime prevalence of venous thromboembolism than were non-migraineurs $(18.9 \%$ v $7.6 \% ; \mathrm{P}<0.001)$, although no association was found with detailed measures of atherosclerosis. ${ }^{33}$ In the context of our analysis, pregnancy itself is a hypercoagulable condition with a high risk of venous thromboembolism, ${ }^{34}$ but no previous studies have investigated the relation between migraines and thromboembolism in pregnant women.

\section{What this study means}

Several possible explanations exist for the association between peripartum migraine and vascular complications during pregnancy. Firstly, for those women with pre-pregnancy migraines, ongoing complications of pregnancy such as vascular diseases may have led to a flare-up of migraine headaches as a result of the stress of being ill. Alternatively, a woman with a history of vascular disease before pregnancy may worry about recurrent complications, which increases stress and the likelihood of having peripartum migraines. However, this seems unlikely because women also may be very ill as a result of the non-vascular diagnoses during pregnancy, but we found no significant association between these conditions and peripartum migraines.

The most logical explanation for the relation between migraine and vascular disease during pregnancy is the existence of overlapping pathophysiological mechanisms in both conditions, compounded by the physiological changes during pregnancy. One study supporting this hypothesis showed that migraineurs with or without aura had increased peripheral and central blood pressure, a decreased diameter and compliance of superficial muscular arteries, and decreased endothelial dilatation response to hyperaemia compared with controls. ${ }^{35}$ These data suggest the existence of a select group of women with migraine who are predisposed to poor compensation to vascular stresses. The physiological changes during pregnancy, such as increased blood volume, stroke volume, and heart rate, and the haemostatic changes in favour of thrombosis may all compound the interactions between migraine and vascular complications. ${ }^{34}$ Regardless of the mechanism, active migraine during pregnancy could be viewed as a potential marker of vascular disease.

Table 5 |Hypertension, with and without gestational hypertension/pre-eclampsia diagnosis codes, and association with migraine codes

\begin{tabular}{|c|c|c|c|}
\hline Type of hypertension & $\begin{array}{l}\text { Migraine rate per } \\
100000^{\star}\end{array}$ & Odds ratio $(95 \% \mathrm{Cl})$ & $\mathrm{p}$ value \\
\hline Hypertension codes (gestational hypertension or pre-eclampsia codes excluded) & 1966 & $11.9(9.2$ to 15.4$)$ & $<0.001$ \\
\hline Gestational hypertension/pre-eclampsia codes (hypertension codes excluded) & 368 & $2.2(2.0$ to 2.4$)$ & $<0.001$ \\
\hline Both gestational hypertension/pre-eclampsia and hypertension codes & 595 & $3.6(3.0$ to 4.3$)$ & $<0.001$ \\
\hline
\end{tabular}


Table 6 |Multivariable logistic regression model for peripartum migraines

\begin{tabular}{lcccc} 
Independent variable & t value & $\begin{array}{c}\text { Standard } \\
\text { error (odds } \\
\text { ratio) }\end{array}$ & Odds ratio (95\% Cl) & P value \\
Age & 11.9 & 0.002 & $1.03(1.02$ to 1.03$)$ & $<0.001$ \\
\hline Pre-eclampsia & 22.9 & 0.083 & $2.29(2.13$ to 2.46$)$ & $<0.001$ \\
\hline All strokes* & 8.86 & 4.61 & $15.05(8.26$ to 27.4$)$ & $<0.001$ \\
\hline $\begin{array}{l}\text { Venous thromboembolism or } \\
\text { pulmonary embolus* }\end{array}$ & 5.11 & 0.74 & $3.23(2.06$ to 7.07$)$ & $<0.001$ \\
\hline $\begin{array}{l}\text { Acute myocardial infarction } \\
\text { or heart disease* }\end{array}$ & 8.06 & 0.2 & $2.11(1.76$ to 2.54$)$ & $<0.001$ \\
\hline \begin{tabular}{l} 
Hypertension* \\
\hline Smoking
\end{tabular} & 14.43 & 1.28 & $8.61(6.43$ to 11.54$)$ & $<0.001$ \\
\hline Diabetes & 17.15 & 0.17 & $2.85(2.53$ to 3.21$)$ & $<0.001$ \\
\hline
\end{tabular}

*Excluding pre-eclampsia diagnoses. patients with intracerebral haemorrhage and $25 \%$ with ischaemic stroke may present with headache. ${ }^{3738}$ In addition, ischaemic strokes can also cause migraine. ${ }^{39}$ Although $78-91 \%$ of patients with cerebral venous thrombosis present with headache,${ }^{40}$ we are uncertain of the specific association because these events were most likely coded as 674.0 (pregnancy related cerebrovascular event). Other complications that often involve headache include severe pre-eclampsia/eclampsia and the accompanying vasculopathy involving vasogenic oedema (posterior reversible encephalopathy syndrome) or postpartum angiopathy (reversible cerebral vasoconstriction occurring postpartum with or without elevated blood pressure). ${ }^{41}$ Confirmation by review of medical records or prospective studies would clarify how often the presenting stroke symptoms lead to misdiagnosis of migraine.

The other limitation relates to establishing cause and effect. For example, on the basis of the available data, the timing of migraine and onset of cardiovascular disease is uncertain. The migraine may have occurred before or after a vascular event, and the ICD-9 codes may have captured prevalent vascular disease before pregnancy. For vascular conditions, such as stroke, myocardial infarction, and venous thromboembolism, however, these are more likely to be coded only for the discharge when they occurred, making multiple admissions for the same patient unlikely. The possibility also exists that once a woman had a stroke, established risk factors in young women, such as migraine, would be more likely to be coded during an admission, strengthening the relation between the two diagnoses. We were also unable to study these associations in non-pregnant women as they were excluded from the analysis. Therefore, we cannot determine whether migraine as a marker for vascular disease during admission to hospital is specific to pregnant women. In general, no cause or effect mechanism can be established on the basis of these results.

Other important data that could not be measured include drugs that women may have been taking to treat cardiovascular risk factors, such as anticoagulants or blood pressure lowering drugs, or migraine specific treatment, such as those used for preventing migraine or treating pain. Use of one or more of these drugs could have influenced the prevalence of cardiovascular disease or migraine during pregnancy. In addition, the cause of stroke could not be determined because results of diagnostic tests were not available in the database. Therefore, the role of patent foramen ovale, a condition associated with both migraine with aura and stroke, cannot be explored in this database.

\section{Conclusions and policy implications}

To summarise, we identified a coexistence of diagnoses of peripartum migraine with vascular diseases and vascular risk factors during pregnancy in a subpopulation of women with active migraine during admission to hospital. Obstetricians, general practitioners, and neurologists should all realise that these results do not 


\section{WHAT IS ALREADY KNOWN ON THIS TOPIC}

The prevalence of migraine during pregnancy is largely unknown and has not been studied in a population based sample

Migraine was significantly associated with diagnoses of stroke in a previous analysis in this population

\section{WHAT THIS STUDY ADDS}

The prevalence of migraine discharge codes was low (185 per 100000 deliveries), but this probably represents only pregnant women with active migraine during hospital admission

Although cause and effect still need to be established, active migraine during pregnancy could be viewed as a marker of vascular diseases, especially ischaemic stroke

apply to every woman with migraine during pregnancy. However, for pregnant women admitted to hospital with active migraines, modifiable cardiovascular risk factors and complications of pregnancy such as pre-eclampsia, should be recognised and treated.

\section{Future research}

The results of this study lay the groundwork for multiple future studies related to migraine and pregnancy. Because of the current lack of information on inpatient ICD-9 coding for migraine, exploring the reliability of these codes from discharge summaries is an important next step. In addition, prospective registries of pregnant women with migraine and vascular diseases (and validation with individual medical records) would help to determine which condition came first and also lead to further hypotheses related to causality. Once causality was established, then interventions such as drug prophylaxis for migraine or vascular diseases during pregnancy could be prescribed to try to reduce the rate of new or recurrent vascular complications.

Contributors: $\mathrm{CDB}$ and $\mathrm{AHJ}$ contributed to the conception and design of the study and to drafting the article, revising it critically for important intellectual content, and final approval of the version to be published. M] contributed to the analysis and interpretation of data, as well as to drafting the article and the final approval of the version to be published. CDB is the guarantor.

Funding: The study was funded in part by the National Institutes of Health (5K12-HD043446-03 and K02-NS058760). The funding agency had no role in the conception or design of the study; the collection, management, analysis, and interpretation of the data; or the preparation, review, or approval of the manuscript. The authors were independent from the funders in all aspects of the study design, analysis of data, and writing of the manuscript.

Competing interests: None declared.

Ethical approval: Not needed.

1 May A, Goadsby P. The trigeminovascular system in humans: pathophysiologic implications for primary headache syndromes of the neural influences on the cerebral circulation. / Cereb Blood Flow Metab 1999;19:115-27.

2 Henry P, Michel P, Brochet B, Dartigues J, Tison S, Salamon R. A nationwide survey of migraine in France: prevalence and clinical features in adults. Cephalalgia 1992;12:229-37.
3 Launer LJ, Terwindt G, Ferrari M. The prevalence and characteristics of migraine in a population-based cohort: the GEM study. Neurology 1999;53:537-42.

4 Chen T-C, Leviton A. Headache recurrence in pregnant women with migraine. Headache 1994;34:107-10.

5 Somerville B. A study of migraine in pregnancy. Neurology 1972;22:824-8.

6 Adeney K, Williams M. Migraine headaches and preeclampsia: an epidemiologic review. Headache 2006;46:794-803.

7 Scher A, Terwindt G, Picavet H, Verschuren W, Ferrari M, Launer LI. Cardiovascular risk factors and migraine: the GEM population-based study. Neurology 2005;64:614-20.

8 Adeney K, Williams M, Miller R, Frederick I, Sorensen T, Luthy D. Risk of preeclampsia in relation to maternal history of migraine headaches. J Matern Fetal Neonatal Med 2005;18:167-72.

9 Facchinetti F, Allais G, D’Amico R, Benedetto C, Volpe A. The relationship between headache and preeclampsia: a case-control study. Eur J Obstet Gynecol Reproduct Biol 2005;121:143-8.

10 Banhidy F, Acs N, Horvath-Puho E, Czeizel A. Pregnancy complications and delivery outcomes in pregnant women with severe migraine. Eur J Obstet Gynecol Reproduct Biol 2007;134:157-63.

11 James AH, Bushnell CD, Jamison MG, Myers ER. Incidence and risk factors for stroke in pregnancy and the puerperium. Obstet Gynecol 2005;106:509-16.

12 James A, Jamison M, Biswas M, Brancazio L, Swamy G, Myers E. Acute myocardial infarction in pregnancy: a United States population-based study. Circulation 2006;113:1564-71.

13 Kurth T, Slomke M, Kase C, Cook N, Lee I-M, Gaziano J, et al. Migraine, headache, and the risk of stroke in women. Neurology 2005;64:1020-6.

14 Kurth T, Gaziano J, Cook N, Logroscino G, Diener H-C, Buring J. Migraine and risk of cardiovascular disease in women. JAMA 2006;296:283-91.

15 Kurth T, Schurks M, Logroscino G, Gaziano J, Buring J. Migraine, vascular risk, and cardiovascular events in women: prospective cohort study. BMJ 2008;337:a636.

16 Overview of the nationwide inpatient sample (NIS) 2000. Rockville, MD: Agency for Healthcare Research and Quality, 2002.

17 Introduction to the nationwide inpatient sample (NIS) 2002. Rockville, MD: Agency for Healthcare Research Quality, 2004.

18 Speirs L, ed. Hospital \& Paylor ICD-9CM. Salt Lake City: Susan PSeare, 1998.

19 Goldstein LB. Accuracy of ICD-9-CM coding for the identification of patients with acute ischemic stroke: effect of modifier codes. Stroke 1998;29:1602-4.

20 Milhaud D, Bogousslavsky J, van Melle G, Liot P. Ischemic stroke and active migraine. Neurology 2001;57:1805-11.

21 Chang C, Donaghy M, Poulter N. Migraine and stroke in young women: a case-control study. BMJ 1999;318:13-8.

22 Tzourio C, Iglesias S, Hubert J-B, Visy J-M, Alperovitch A, Tehindrazanarivelo A, et al. Migraine and risk of ischaemic stroke: a case-control study. BMJ 1993;307:289-92.

23 Tzourio C, Tehindrazanarivelo A, Iglesias S, Alperovitch A, Chedru F, d'Anglejan-Chatillon J, et al. Case-control study of migraine and risk of ischaemic stroke in young women. BMJ 1995;310:830-3.

24 Schwaag S, Nabavi D, Frese A, Husstedt I-W, Evers S. The association between migraine and juvenile stroke: a case-control study. Headache 2003;43:90-5.

25 Etminan M, Takkouche B, Caamano I, Samii A. Risk of ischaemic stroke in people with migraine: systematic review and meta-analysis of observational studies. BMJ 2005:330;63.

26 Chen T, Leviton A, Edelstein S, Ellenberg J. Migraine and other diseases in women of reproductive age: the influence of smoking on observed associations. Arch Neurol 1987;44:1024-8.

27 Mitchell P, Cumming R, Wang J, Smith W, Currie J. Prevalence and vascular associations with migraine in older Australians. Aust NZJ Med 1998;28:627-32.

28 Rose K, Carson AP, Sanford CP, Stang P, Brown C, Folsom A, Szklo M. Migraine and other headaches: associations with Rose angina and coronary heart disease. Neurology 2004;63:2233-9.

29 Sternfeld B, Stang P, Sidney S. Relationship of migraine headaches to experience of chest pain and subsequent risk for myocardial infarction. Neurology 1995;45:2135-42.

30 Moore M, Redman C. Case-control study of severe pre-eclampsia of early onset. BMJ 1983;287:580-3.

31 Silvestrini M, Matteis M, Troisi E, Cupini L, Zaccari G, Bernardi G. Migrainous stroke and the antiphoshpholipid antibodies. Eur Neurol 1994;34:316-9.

32 Crassard I, Conard J, Bousser M-G. Migraine and haemostasis. Cephalalgia 2001;21:630-6.

33 Schwaiger J, Kiechl S, Stockner H, Knoflach M, Werner P, Rungger G, et al. Burden of atherosclerosis and risk of venous thromboembolism in patients with migraine. Neurology 2008;71:937-43.

34 Lockwood C. Inherited thrombophilias in pregnant patients: detection and treatment paradigm. Obstet Gynecol 2002;99:333-41. 
35 Vanmolkot F, Van Bortel L, de Hoon J. Altered arterial function in migraine of recent onset. Neurology 2007;68:1563-70.

36 MacClellan LR, Giles W, Cole J, Wozniak M, Stern B, Mitchell BD, et al. Probable migraine with visual aura and risk of ischemic stroke: the stroke prevention in young women study. Stroke 2007;38:2407-8.

37 Vestergaard K, Andersen G, Nielsen M, Jensen T. Headache in stroke. Stroke 1993;24:1621-4.

38 Gorelick P, Hier D, Caplan L, Langenberg P. Headache in acute cerebrovascular disease. Neurology 1986;36:1445-50.
39 Olesen J, Friberg L, Olsen T, Andersen A, Lassen N, Hansen P, et al. Ischaemia-induced (symptomatic) migraine attacks may be more frequent than migraine-induced ischaemic insults. Brain 1993;116:187-202.

40 Bousser M-G, Barnett HJ. Cerebral venous thrombosis. In: Barnett HJ, Mohr J, Stein BM, Yatsu FM, eds. Stroke: pathophysiology, diagnosis, and management. 3rd ed. New York: Churchill Livingstone, 1998:623-47. 